\title{
The estimation of generalized extreme value models from choice-based samples
}

\author{
M. Bierlaire, D. Bolduc and D. McFadden
}

Transport and Mobility Laboratory, EPFL

Département d'économique, Université Laval, Québec

Econometrics Laboratory, University of California, Berkeley 


\section{Outline}

- Introduction

- Sampling

- Estimation

- Multivariate (generalized) extreme value models

- Illustrations 


\section{Introduction}

- Sampling is never random in practice

- Choice-based samples are convenient in transportation analysis

- Estimation is an issue

- Main references:

- Manski and Lerman (1977)

- Manski and McFadden (1981)

- Cosslett (1981)

- Ben-Akiva and Lerman (1985) 


\section{Sampling: context}

- Discrete choice model, $J$ alternatives

- Independent variables: $x$

- Dependent variable (choice): $i$

- Model:

$$
\operatorname{Pr}(i \mid x, \theta)=P(i \mid x, \theta)
$$

- Unknown parameters: $\theta$

- Joint distribution of $(i, x)$ in the population

$$
\operatorname{Pr}(i, x \mid \theta)=P(i \mid x, \theta) p(x) .
$$




\section{Sampling: stratification}

- Population partitioned into $G$ groups

- Individuals randomly selected within each group

- Population size: $N_{P}$

- \% of ind. from group $g$ in population: $W_{g}$

- Sample size: $N_{s}$

- \% of ind. from group $g$ in sample: $H_{g}$

- Probability to be in the sample: $r_{g}=\frac{H_{g} N_{s}}{W_{g} N_{P}}$. 


\section{Sampling strategies}

SRS Simple random sampling

- Only one group.

- $H_{g}=W_{g}$,

- $r_{g}=r=N_{s} / N_{P}$.

XSS Exogenous stratified sampling

- Groups characterized by $x$

- $W_{g}=\int_{x \in X_{g}} p(x) d x$

- $r_{g}$ does not depend on $\theta$. 


\section{Sampling strategies}

ESS Endogenous stratified sampling

- Groups characterized by $i$

- $W_{g}$ does not simplify

- $r_{g}$ depends on $\theta$

XESS Exogenous and endogenous stratified sampling

- Groups characterized both by $x$ and $i$

- $W_{g}$ does not simplify

- $r_{g}$ depends on $\theta$ 


\section{Sampling of alternatives}

- Analyze choice as if limited to $\mathcal{B} \subseteq \mathcal{C}$

- $\mathcal{B}$ is drawn with prob. $\pi(\mathcal{B} \mid i, x)$

- Positive conditioning property:

$$
\pi(\mathcal{B} \mid i, x)>0 \Rightarrow \pi(\mathcal{B} \mid j, x)>0 \quad \forall j \in \mathcal{B} .
$$

- Appropriate sampling:

$$
\pi(\mathcal{B} \mid i, x)>0 \Rightarrow r_{g(i, x)}>0
$$




\section{Sampling}

Probability that a population member with configuration $(i, x)$ is sampled, and is assigned the truncated choice set $\mathcal{B}$ :

$$
R(i, x, \mathcal{B}, \theta)=\operatorname{Pr}(s, \mathcal{B} \mid i, x, \theta)=r_{g(i, x)}(\theta) \pi(\mathcal{B} \mid i, x) .
$$




\section{Estimation}

\section{Conditional Maximum Likelihood (CML)}

Estimator

$$
\begin{aligned}
& \max _{\theta} \mathcal{L}(\theta)=\sum_{n=1}^{N} \ln \operatorname{Pr}\left(i_{n} \mid x_{n}, \mathcal{B}_{n}, s, \theta\right) \\
& =\sum_{n=1}^{N} \ln \frac{R\left(i_{n}, x_{n}, \mathcal{B}_{n}, \theta\right) P\left(i_{n} \mid x_{n}, \theta\right)}{\sum_{j \in \mathcal{B}_{n}} R\left(j, x_{n}, \mathcal{B}_{n}, \theta\right) P\left(j \mid x_{n}, \theta\right)}
\end{aligned}
$$

In practive, $R\left(i_{n}, x_{n}, \mathcal{B}_{n}, \theta\right)$ cannot be computed, namely because it requires $p(x)$ 


\section{Estimation}

Assume that $R(i, x, \mathcal{B}, \theta)$ can be written as

$$
R(i, x, \mathcal{B}, \theta)=Q(i, x, \mathcal{B}) S(i, x, \mathcal{B}, \theta) .
$$

Pseudo-likelihood function

$$
\begin{aligned}
& \widehat{\mathcal{L}}=\sum_{n=1}^{N} Q\left(i_{n}, x_{n}, \mathcal{B}_{n}\right)^{-1} \ln \frac{S\left(i_{n}, x_{n}, \mathcal{B}_{n}, \theta\right) P\left(i_{n} \mid x_{n}, \theta\right)}{\sum_{j \in \mathcal{B}_{n}} S\left(j, x_{n}, \mathcal{B}_{n}, \theta\right) P\left(j \mid x_{n}, \theta\right)} \\
& \text { - } Q=1 \text { : CML by Manski \& McFadden (1981) } \\
& \text { - } S=1 \text { : WESML by Manski \& Lerman (1977) }
\end{aligned}
$$




\section{Estimation of MEV models}

- Let $G$ be the generating function of a MEV model

- Let

$$
G_{i}(x, \beta, \gamma)=\frac{\partial G}{\partial e^{V_{i}(x, \beta)}}\left(e^{V_{1}(x, \beta)}, \ldots, e^{V_{J}(x, \beta)} ; \gamma\right) .
$$

- The main term in the $\mathrm{CML}$ formulation is:

$$
\frac{S(i, x, \mathcal{B}, \theta) P(i \mid x, \theta)}{\sum_{j \in \mathcal{B}} S(j, x, \mathcal{B}, \theta) P(j \mid x, \theta)}=\frac{e^{V_{i}(\beta)+\ln G_{i}(x, \beta, \gamma)+\ln S(i, x, \mathcal{B}, \theta)}}{\sum_{j \in \mathcal{B}} e^{V_{j}(\beta)+\ln G_{j}(x, \beta, \gamma)+\ln S(j, x, \mathcal{B}, \theta)}}
$$




\section{Estimation of MEV models}

- The term needed for CML is MNL-like

- Case of MNL model: $G_{i}=0$.

$$
\frac{S(i, x, \mathcal{B}, \theta) P(i \mid x, \theta)}{\sum_{j \in \mathcal{B}} S(j, x, \mathcal{B}, \theta) P(j \mid x, \theta)}=\frac{e^{V_{i}(\beta)+\ln S(i, x, \mathcal{B}, \theta)}}{\sum_{j \in \mathcal{B}} e^{V_{j}(\beta)+\ln S(j, x, \mathcal{B}, \theta)}} .
$$

- Well-known result: if ESML is used, only constants are biased

- Question: does this generalize to all MEV?

- Answer: not quite... 


\section{Estimation of MEV models}

- The $V$ 's are shifted in the main formula

$$
\frac{e^{V_{i}(\beta)+\ln G_{i}(x, \beta, \gamma)+\ln S(i, x, \mathcal{B}, \theta)}}{\sum_{j \in \mathcal{B}} e^{V_{j}(\beta)+\ln G_{j}(x, \beta, \gamma)+\ln S(j, x, \mathcal{B}, \theta)}} .
$$

- ... but not in the $G_{i}$

$$
G_{i}(x, \beta, \gamma)=\frac{\partial G}{\partial e^{V_{i}(x, \beta)}}\left(e^{V_{1}(x, \beta)}, \ldots, e^{V_{J}(x, \beta)} ; \gamma\right)
$$

- ESML will not produce consistent estimates on non-MNL MEV models. 


\section{Estimation of MEV models}

$$
\frac{e^{V_{i}(\beta)+\ln G_{i}(x, \beta, \gamma)+\ln S(i, x, \mathcal{B}, \theta)}}{\sum_{j \in \mathcal{B}} e^{V_{j}(\beta)+\ln G_{j}(x, \beta, \gamma)+\ln S(j, x, \mathcal{B}, \theta)}} .
$$

- New idea: estimate $\ln S(i, x, \mathcal{B}, \theta)$ from data

- Cannot be done with classical software

- But easy to implement due to the MNL-like form 


\section{Illustration}

- Pseudo-synthetic data

- Data base: SP mode choice for future highspeed train in Switzerland (Swissmetro)

- Alternatives:

1. Regular train (TRAIN),

2. Swissmetro (SM), the future high speed train,

3. Driving a car (CAR).

- Generation of a synthetic population of 507600 individuals 


\section{Illustration}

- Attributes are random perturbations of actual attributes

- Assumed true choice model: NL

\begin{tabular}{rrrrr} 
& & \multicolumn{3}{c}{ Alternatives } \\
\cline { 3 - 5 } Param. & Value & TRAIN & SM & CAR \\
\hline ASC_CAR & -0.1880 & 0 & 0 & 1 \\
ASC_SM & 0.1470 & 0 & 1 & 0 \\
B_TRAIN_TIME & -0.0107 & travel time & 0 & 0 \\
B_SM_TIME & -0.0081 & 0 & travel time & 0 \\
B_CAR_TIME & -0.0071 & 0 & 0 & travel time \\
B_COST & -0.0083 & travel cost & travel cost & travel cost \\
\hline
\end{tabular}




\section{Illustration}

- Nesting structure:

\begin{tabular}{lrrrr} 
& $\mu_{m}$ & TRAIN & SM & CAR \\
\hline NESTA & 2.27 & 1 & 0 & 1 \\
NESTB & 1.0 & 0 & 1 & 0
\end{tabular}




\section{Illustration}

- 100 samples drawn from the population

\begin{tabular}{rrrrrr} 
Strata & $W_{g} N_{P}$ & $W_{g}$ & $H_{g}$ & $H_{g} N_{s}$ & $R_{g}$ \\
\hline TRAIN & 67938 & $13.4 \%$ & $60 \%$ & 3000 & $4.42 \mathrm{E}-02$ \\
SM & 306279 & $60.3 \%$ & $20 \%$ & 1000 & $3.26 \mathrm{E}-03$ \\
CAR & 133383 & $26.3 \%$ & $20 \%$ & 1000 & $7.50 \mathrm{E}-03$ \\
\hline Total & 507600 & 1 & 1 & 5000 &
\end{tabular}

- Estimation of 100 models

- Empirical mean and std dev of the estimates 


\section{Illustration}

\begin{tabular}{r|r|rrr|rrr} 
& \multicolumn{3}{c}{ ESML } & \multicolumn{3}{c}{ New estimator } \\
& True & Mean & $t$-test & Std. dev. & Mean & $t$-test & Std. dev. \\
\hline ASC_SM & 0.1470 & -2.2479 & -25.4771 & 0.0940 & -2.4900 & -23.9809 & 0.1100 \\
ASC_CAR & -0.1880 & -0.8328 & -7.3876 & 0.0873 & -0.1676 & 0.1581 & 0.1292 \\
BCOST & -0.0083 & -0.0066 & 2.6470 & 0.0007 & -0.0083 & 0.0638 & 0.0008 \\
BTIME_TRAIN & -0.0107 & -0.0094 & 1.4290 & 0.0009 & -0.0109 & -0.1774 & 0.0009 \\
BTIME_SM & -0.0081 & -0.0042 & 3.1046 & 0.0013 & -0.0080 & 0.0446 & 0.0014 \\
BTIME_CAR & -0.0071 & -0.0065 & 0.9895 & 0.0007 & -0.0074 & -0.3255 & 0.0007 \\
NestParam & 2.2700 & 2.7432 & 1.7665 & 0.2679 & 2.2576 & -0.0609 & 0.2043 \\
\hline S_SM_Shifted & -2.6045 & & & & & & \\
S_CAR_Shifted & -1.7732 & & & & -1.7877 & -0.0546 & 0.2651 \\
ASC_SM+S_SM & -2.4575 & & & & -2.4900 & -0.2958 & 0.1100
\end{tabular}




\section{Conclusions}

- Except in very specific cases, ESML provides biased estimated for non-MNL MEV models

- Due to the MNL-like form of the MEV model, a new simple estimator has been proposed

- It allows to estimate selection bias from the data 